Article

\title{
The Harmonic Mapping Whose Hopf Differential Is a Constant
}

\section{Liang Shen 1}

School of Mathematics and Statistics, Beijing Institute of Technology, Beijing 100081, China; shenl@bit.edu.cn

Received: 16 July 2020; Accepted: 3 August 2020; Published: 7 August 2020

\begin{abstract}
Suppose that $h(z)$ is a harmonic mapping from the unit disk $\mathbb{D}$ to itself with respect to the hyperbolic metric. If the Hopf differential of $h(z)$ is a constant $c>0$, the Beltrami coefficient $\mu(z)$ of $h(z)$ is radially symmetric and takes the maximum at $z=0$. Furthermore, the mapping $\gamma: c \rightarrow \mu(0)$ is increasing and gives a homeomorphism from $(0,+\infty)$ to $(0,1)$.
\end{abstract}

Keywords: harmonic mapping; Hopf differential; Beltrami coefficient

\section{Introduction}

Let $h(z)$ be an orientation preserving diffeomorphism from the unit disk $\mathbb{D}$ to itself. The mapping $h(z)$ is harmonic with respect to the hyperbolic metric on $\mathbb{D}$ if it satisfies the Euler-Lagrange equation

$$
h_{z \bar{z}}+2 \frac{\rho_{z}(h)}{\rho(h)} h_{z} h_{\bar{z}}=0,
$$

where $\rho^{2}(z)|d z|^{2}=\frac{4}{\left(1-|z|^{2}\right)^{2}}|d z|^{2}$ is the hyperbolic metric on $\mathbb{D}$.

The harmonic mapping is closed related to the quasiconformal mapping and Teichmüller theory. For example, the Schoen conjecture, proved by Li and Tam in [1,2] and Markovic in [3], says that for every quasisymmetric homeomorphism on the unit circle $\mathbb{T}$, there exists a unique harmonic extension to the interior $\mathbb{D}$.

The Hopf differential $\phi(z)=\rho^{2}(h) h_{z} \overline{h_{\bar{z}}}$ of a harmonic mapping $h(z)$ is always holomorphic by (1). Given a Hopf differential, the existence and uniqueness of the harmonic mapping is established by Wan in [4]. Also in [4], it is proved that a harmonic mapping is quasiconformal if and only if the Bers norm of the Hopf differential is bounded. Especially if the Hopf differential is constant, the harmonic mapping is quasiconformal. In the paper, we consider such mappings and prove the following result.

Theorem 1. Suppose $h(z)$ is a harmonic mapping whose Hopf differential is a constant $c>0$. The Beltrami coefficient $\mu(z)$ of $h(z)$ is a radially symmetric real function and takes the maximum at $z=0$. The mapping $\gamma: c \rightarrow \mu(0)$ is increasing and gives a homeomorphism from $(0,+\infty)$ to $(0,1)$.

Let $h_{c}(z)$ be a harmonic mapping with the Hopf differential $c \phi(z)$ between compact surfaces. In [5], Wolf proved that the Beltrami coefficient $\mu_{c}(z)$ of $h_{c}(z)$ is an increasing function of $c>0$, which is used to give a compactification of Teichmüller space. But the method can not be generalized to the harmonic mapping on the unit disk. In the proof of Theorem 1, we use the similar method as in $[4,6]$, i.e., the generalized maximum principle of Cheng and Yau [7].

In the following, the Laplace-Beltrami operator is denoted by $\Delta_{g}$ on a Riemannian manifold $(M, g)$. For a conformal metric $\sigma|d z|^{2}$ on the unit disk $\mathbb{D}$, the Laplace-Beltrami operator has the following form

$$
\Delta_{\sigma}=\frac{\Delta}{\sigma}
$$


where $\Delta=\frac{\partial^{2}}{\partial x^{2}}+\frac{\partial^{2}}{\partial y^{2}}$ is the Laplace operator.

\section{The Absolute Value of the Beltrami Coefficient Is Radially Symmetric}

First we give some known facts about harmonic mappings.

Let $h(z)$ be a harmonic mapping with respect to the hyperbolic metric on the unit disk $\mathbb{D}$. Let $\phi(z)$ be the Hopf differential and $\mu(z)$ be the Beltrami coefficient of $h(z)$. The Bochner formula gives:

$$
\Delta_{\rho^{2}} \log |\partial h|=|\partial h|^{2}-|\bar{\partial} h|^{2}-1, \quad \Delta_{\rho^{2}} \log |\bar{\partial} h|=-\left(|\partial h|^{2}-|\bar{\partial} h|^{2}\right)-1
$$

where $\partial h=\frac{\rho(h(z))}{\rho(z)} h_{z}, \bar{\partial} h=\frac{\rho(h(z))}{\rho(z)} h_{\bar{z}}$. By the first equality, we have

$$
\Delta v=e^{2 v}-|\phi|^{2} e^{-2 v} \text { and } \Delta v>0 .
$$

where $e^{2 v}=\rho^{2}(h(z))\left|h_{z}\right|^{2}$. The Beltrami coefficient $\mu(z)$ satisfies that

$$
\mu(z)=\frac{h_{\bar{z}}}{h_{z}}=\frac{\rho^{2}(h(z)) \overline{h_{z}} h_{\bar{z}}}{\rho^{2}(h(z))\left|h_{z}\right|^{2}}=\frac{\bar{\phi}}{e^{2 v}} .
$$

On the other hand, the following theorem was proved by Wan in [4].

Theorem 2 (Theorem 12, [4]). For any holomorphic function $\phi(z)$ on $\mathbb{D}$, there exists a unique smooth function $v(z)$ such that

1. $\Delta v=e^{2 v}-|\phi|^{2} e^{-2 v}>0$,

2. $e^{2 v}|d z|^{2}$ is complete.

Theorem 2 gives a way to construct a harmonic mapping from a Hopf differential. From the Hopf differential $\phi$ one has the Beltrami coefficient $\mu(z)$, then solve the Beltrami equation to obtain the harmonic mapping.

In the following, we discuss the harmonic mappings which has special Hopf differentials. First we show for a class of Hopf differentials, the metric $e^{2 v}|d z|^{2}$ is radially symmetric.

Lemma 1. Suppose the Hopf differential $\phi(z)$ is $c z^{n}$ where $c \in \mathbb{C}$ and $n=0,1,2, \cdots$. The metric $e^{2 v}|d z|^{2}$ is radially symmetric, i.e., $v\left(e^{i \theta} z\right)=v(z)$.

Proof. For the Hopf differential $\phi=c z^{n}$, by Theorem 2, there is a unique smooth function $v(z)$ such that

1. $\Delta v=e^{2 v}-|\phi|^{2} e^{-2 v}>0$,

2. $e^{2 v}|d z|^{2}$ is complete.

Let $w=e^{i \theta} z$ and $v_{1}(z)=v\left(e^{i \theta} z\right)$. Since

$$
\Delta v(w)=e^{2 v(w)}-|\phi(w)|^{2} e^{-2 v(w)}
$$

and

$$
\Delta v(w)=\left.4 \frac{\partial^{2} v_{2}}{\partial_{w} \partial_{\bar{w}}}\right|_{w=e^{i \theta} z}=4 \frac{\partial^{2}}{\partial_{z} \partial_{\bar{z}}} v_{2}\left(e^{i \theta} z\right)=\Delta\left(v\left(e^{i \theta} z\right)\right),
$$

we have

$$
\Delta v_{1}=e^{2 v_{1}}-\left|c z^{n}\right|^{2} e^{-2 v_{1}} .
$$

By the uniqueness of the solution, the function $v_{1}(z)$ must be $v(z)$. So $v(z)$ is radially symmetric. 
In particular, if the Hopf differential is a constant $c \in \mathbb{C}$, the metric $e^{2 v}|d z|^{2}$ is radially symmetric. If $\phi_{1}(z)$ and $\phi_{2}(z)$ are two holomorphic functions which satisfy that $\phi_{1}(z)=$ $e^{i \alpha} \phi_{2}(z)$, the corresponding $v_{1}(z)$ and $v_{2}(z)$ satisfy $v_{1}(z)=v_{2}(z)$ by the uniqueness. As a result, the corresponding Beltrami coefficients satisfies that $\mu_{1}(z)=e^{-i \alpha} \mu_{2}(z)$. So we only need consider the Hopf differential is a positive number $c>0$. In these cases, the Beltrami coefficients are real functions. In the following lemma, we consider the maximum of $|\mu(z)|$.

Lemma 2. Let $\phi(z)$ be Hopf differential of a harmonic mapping $h(z)$ and $\mu(z)$ be the Beltrami coefficient. If $\phi(z)$ has no zeros, the function $|\mu(z)|$ has no minimum in $\mathbb{D}$.

Proof. Since $|\phi(z)|>0$ and $\mu=\frac{\bar{\phi}}{e^{2 v}}$, the Beltrami coefficient $\mu(z)$ satisfies that $|\mu(z)|>0$. The Bochner formula

$$
\Delta_{\rho^{2}} \log |\partial h|=|\partial h|^{2}-|\bar{\partial} h|^{2}-1, \quad \Delta_{\rho^{2}} \log |\bar{\partial} h|=-\left(|\partial h|^{2}-|\bar{\partial} h|^{2}\right)-1
$$

hold for every $z \in \mathbb{D}$. Then we have

$$
\Delta_{\rho^{2}} \log |\mu(z)|=-2|\partial h|^{2}\left(1-|\mu(z)|^{2}\right),
$$

which implies that $\Delta \log |\mu(z)|<0$ holds for every $z \in \Delta$. So $\log |\mu(z)|$ is a superharmonic function on $\mathbb{D}$, which implies that $\log |\mu(z)|$ can not obtain the minimum in $\mathbb{D}$. Then the function $|\mu(z)|$ has no minimum in $\mathbb{D}$.

If the Hopf differential of a harmonic mapping $h(z)$ is a constant $c \in \mathbb{C}$, the absolute value of the Beltrami coefficient $\mu$ is radially symmetric by Lemma 1 . Since $\lim _{|z| \rightarrow 1}|\mu(z)|=0$, the function $|\mu(z)|$ has an absolute maximum in $\mathbb{D}$. If $|\mu(z)|$ obtains a maximum at $z_{0} \neq 0,|\mu(z)|$ also obtains a maximum at $e^{i \theta} z_{0}$. As a result, $|\mu(z)|$ have a minimum in $|z|<\left|z_{0}\right|$, which contradicts to Lemma 2. So $|\mu(z)|$ takes the absolute maximum only at $z=0$. It is the context of the following lemma. Here we give a direct proof.

Lemma 3. Suppose the Hopf differential of a harmonic mapping $h(z)$ is a constant $c \in \mathbb{C}$. The maximum of $|\mu(z)|$ is obtained at $z=0$.

Proof. From (2), the following equality holds

$$
\Delta \log |\mu(z)|=-2 \rho^{2}(h(z))\left|h_{z}\right|^{2}\left(1-|\mu(z)|^{2}\right),
$$

which yields

$$
\Delta \log |\mu(z)|=-2 e^{2 v}\left(1-|\mu(z)|^{2}\right)
$$

Then we have

$$
-\frac{1}{2} \Delta \log |\mu(z)|=\frac{|\phi|}{|\mu|}\left(1-|\mu(z)|^{2}\right) .
$$

Define a function $\tau(z)=\frac{1}{2} \log \frac{1}{|\mu|}>0$. The above equality becomes

$$
\Delta \tau=\left(e^{2 \tau}-e^{-2 \tau}\right)|\phi|
$$

Recall that the Laplace operator in polar coordinates is given by

$$
\Delta=\frac{\partial^{2}}{\partial r^{2}}+\frac{1}{r} \frac{\partial}{\partial r}+\frac{1}{r^{2}} \frac{\partial^{2}}{\partial \theta^{2}} .
$$


Since $\tau(z)$ is radially symmetric, it is a function of $r=|z|$ and satisfies

$$
\tau^{\prime \prime}(r)+\frac{1}{r} \tau^{\prime}(r)=|c|\left(e^{2 \tau}-e^{-2 \tau}\right)>0
$$

The left side is equal to $\frac{\left(r \tau^{\prime}(r)\right)^{\prime}}{r}$, so $\left(r \tau^{\prime}(r)\right)^{\prime}>0$, which implies $r \tau^{\prime}(r)$ is a strictly increasing function of $r>0$. The function $\tau(x)$ on the line $(-1,1)$ is an even function of $x$. It satisfies that $\tau^{\prime}(0)=0$.

So $r \tau^{\prime}(r)$ takes value 0 at $z=0$. As a result, $r \tau^{\prime}(r)>0$, which means $\tau(r)$ is a strictly increasing function. Then $|\mu(z)|$ takes maximum at $z=0$.

Finally we give a geometric description of a harmonic mapping whose Hopf differential is a constant.

Lemma 4. Suppose the Hopf differential of a harmonic mapping $h(z)$ is a constant $c>0$. The following properties hold:

(1). Suppose the mapping $h(z)$ satisfies that $h(0)=0, h(1)=1$, then $h(z)$ maps the $x$-axis onto $x$-axis and the $y$-axis onto $y$-axis.

(2). Let $\gamma_{1}$ and $\gamma_{2}$ be a pair of intersecting lines in the disk $\mathbb{D}$ which are horizontal and vertical respectively. Then the curves $h\left(\gamma_{1}\right)$ and $h\left(\gamma_{2}\right)$ are orthogonal.

Proof. (1). The mapping $H(z)=\overline{h(\bar{z})}$ is also harmonic and has the same Hopf differential with $h(z)$. Since $H(z)$ keeps 0,1 fixed, we have $H(z)=h(z)$. So $h(z)$ maps the $x$-axis onto $x$-axis. Similarly we have $h(z)=-h(-z)$, which implies that $h(z)$ maps the $y$-axis onto $y$-axis.

Now we prove (2). First assume that the Hopf differential of a harmonic mapping $h(z)=$ $u(x, y)+i v(x, y)$ is a real number. By the following calculations,

$$
\begin{gathered}
h_{z}=\frac{1}{2}\left(h_{x}-i h_{y}\right)=\frac{1}{2}\left(u_{x}+v_{y}-i\left(u_{y}-v_{x}\right)\right), \\
h_{\bar{z}}=\frac{1}{2}\left(h_{x}+i h_{y}\right)=\frac{1}{2}\left(u_{x}-v_{y}+i\left(u_{y}+v_{x}\right)\right), \\
\operatorname{Im}(\phi)=\rho^{2}(h) \operatorname{Im}\left(h_{z} \overline{h_{\bar{z}}}\right)=-\frac{1}{2} \rho^{2}(h)\left(u_{x} u_{y}+v_{x} v_{y}\right),
\end{gathered}
$$

we find $u_{x} u_{y}+v_{x} v_{y}=0$, which implies that the curves $h\left(\gamma_{1}\right)$ and $h\left(\gamma_{2}\right)$ are orthogonal.

\section{The Value $\mu_{c}(0)$ Is Strictly Increasing}

Let's recall the following lemma of Cheng and Yau [8].

Lemma 5. Let $(M, g)$ be a complete manifold with the Ricci curvature bounded below by some constant. Suppose $\alpha \in C^{2}(M)$ satisfies

$$
\Delta_{g} \alpha \geq f(\alpha),
$$

where $f(x)$ is a continuous function which is positive and non-decreasing near $\infty$ and satisfies that

$$
\int_{p}^{\infty} d t\left(\int_{q}^{t} f(x) d x\right)^{-\frac{1}{2}}<\infty
$$

for some constants $p>q$. Then $\alpha$ is bounded from above.

The following maximum principle given by Omori [9] and Yau [7] is well-known. 
Lemma 6 (Omori-Yau maximum principle). Let $(M, g)$ be a complete manifold with the Ricci curvature of $M$ bounded below by some constant. If $\alpha \in C^{2}(M)$ is bounded from above, then for any $\varepsilon>0$, there is a point $x_{k} \in M$ such that

$$
\alpha\left(x_{k}\right)>\sup _{\mathbb{D}} \alpha-\varepsilon, \quad\left|\nabla_{g} \alpha\left(x_{k}\right)\right|<\varepsilon, \quad \Delta_{g} \alpha\left(x_{k}\right)<\varepsilon .
$$

Let $\phi$ be a Hopf differential and $e^{2 v}|d z|^{2}$ be the corresponding complete metric in Theorem 2. The Gauss(Ricci) curvature of $e^{2 v}|d z|^{2}$ is

$$
K=-\frac{\Delta v}{e^{2 v}}=|\mu(z)|^{2}-1
$$

Now we can prove the following lemma.

Lemma 7. Let $\phi_{1}$ and $\phi_{2}$ be Hopf differentials of harmonic mappings $h_{1}, h_{2}$ on $\mathbb{D}$ respectively, which satisfy that $\left|\phi_{1}(z)\right|=c>0$ and $\left|\phi_{1}\right| \leq\left|\phi_{2}\right|$. Then the Beltrami coefficients $\mu_{1}$ and $\mu_{2}$ satisfy that $\left|\mu_{1}\right| \leq\left|\mu_{2}\right|$.

Proof. Let $\tau_{i}(z)=\frac{1}{2} \log \frac{1}{\left|\mu_{i}(z)\right|}(i=1,2)$ as before. We have $\tau_{i}(z)>0$ since $0<\left|\mu_{i}(z)\right|<1$. Define $\eta(z)=\tau_{2}(z)-\tau_{1}(z)$. Since

$$
\Delta \tau_{i}=\left(e^{2 \tau_{i}}-e^{-2 \tau_{i}}\right)\left|\phi_{i}\right| \quad(i=1,2),
$$

we have

$$
\begin{aligned}
\Delta \eta & =\left|\phi_{2}\right|\left(e^{2 \tau_{2}}-e^{-2 \tau_{2}}\right)-\left|\phi_{1}\right|\left(e^{2 \tau_{1}}-e^{-2 \tau_{1}}\right) \\
& \geq c\left(e^{2 \tau_{2}}-e^{-2 \tau_{2}}\right)-c\left(e^{2 \tau_{1}}-e^{-2 \tau_{1}}\right) .
\end{aligned}
$$

By multiplying by $e^{-2 \tau_{1}}$, the inequality becomes

$$
e^{-2 \tau_{1}} \Delta \eta \geq c\left(e^{2 \eta}-e^{-4 \tau_{1}} e^{-2 \eta}-1+e^{-4 \tau_{1}}\right) .
$$

Since $e^{-4 \tau_{1}}<1$, we have

$$
e^{-2 \tau_{1}} \Delta \eta \geq c\left(e^{2 \eta}-e^{-2 \eta}-1\right)
$$

Let $f(x)=c\left(e^{2 x}-e^{-2 x}-1\right)$, which is positive and non-decreasing as $x$ tends to $+\infty$. The function $f(x)$ satisfies that

$$
\int_{p}^{\infty} d t\left(\int_{q}^{t} f(x) d x\right)^{-\frac{1}{2}}<\infty
$$

for some constants $p>q$. The inequality (4) can be written as

$$
\Delta_{\rho_{1}} \eta \geq f(\eta)
$$

The metric $\rho_{1}=e^{2 \tau_{1}}$ is complete on $\mathbb{D}$ since $e^{2 \tau_{1}}=\frac{1}{\left|\mu_{1}\right|}=\frac{e^{2 v_{1}}}{c}$.

The Gauss curvatures of $e^{2 \tau_{1}}$ and $e^{2 v_{1}}$ satisfy

$$
K\left(e^{2 \tau_{1}}\right)=c K\left(e^{2 v_{1}}\right) .
$$

Since $K\left(e^{2 v_{1}}\right)=\left|\mu_{1}\right|^{2}-1$ has a lower bound, the Gauss curvature of $e^{2 \tau_{1}}$ also has a lower bound. Then we can use Lemma 5 to conclude that $\eta$ is bounded from above. By Lemma 6 , there is a sequence of points $x_{k}$ in $\mathbb{D}$, such that

$$
\lim _{k \rightarrow \infty} \eta\left(x_{k}\right)=\sup _{\mathbb{D}} \eta(=\bar{\eta}), \quad \lim _{k \rightarrow \infty} \Delta_{\rho_{1}} \eta\left(x_{k}\right) \leq 0 .
$$


Since the sequence $\left\{e^{-4 \tau_{1}\left(x_{k}\right)}\right\}$ is bounded, we can take a subsequence such that $\lim _{k \rightarrow \infty} e^{-4 \tau_{1}\left(x_{k}\right)}=$ $\xi(\leq 1)$. By taking limits on two sides of (3), we get

$$
0 \geq c\left(e^{2 \bar{\eta}}-\xi e^{-2 \bar{\eta}}-1+\xi\right) .
$$

Let

$$
g(t)=c\left(e^{2 t}-\xi e^{-2 t}-1+\xi\right) .
$$

It is an increasing function of $t$ and $g(0)=0$. Then $g(\bar{\eta}) \leq 0$ implies that $\bar{\eta} \leq 0$. So we have $\eta(z) \leq 0$ and $\tau_{2} \leq \tau_{1}$.

To prove that $\gamma$ is strictly increasing, we use the following lemma of Heinz [10].

Lemma 8. Let $\alpha(z)$ be a non-negative $C^{2}$-function on $\mathbb{D}(R)$ which satisfies

$$
\Delta \alpha \leq C \alpha, \quad C>0
$$

Then for $0 \leq r<R$,

$$
\frac{1}{\pi r^{2}} \iint_{\mathbb{D}(r)} \alpha(z) d \sigma \leq e^{\frac{C r^{2}}{4}} \alpha(0)
$$

Lemma 8 has the following corollary. It can also be proved directly. We sketch a proof for the sake of completeness.

Corollary 1. Suppose $\alpha(z) \geq 0$ is defined on $\mathbb{D}(R)$ and $\alpha(0)=0$. If

$$
\Delta \alpha \leq C \alpha, \quad C>0
$$

then $\alpha(z) \equiv 0$.

Proof. Define

$$
v(r)=\frac{1}{2 \pi} \int_{0}^{2 \pi} \alpha\left(r e^{i \theta}\right) d \theta, \quad V(r)=\int_{0}^{r} v(\rho) d \rho .
$$

By Green's theorem,

$$
v^{\prime}(r)=\frac{1}{2 \pi} \frac{d}{d r} \int_{0}^{2 \pi} \alpha\left(r e^{i \theta}\right) d \theta=\frac{1}{2 \pi r} \iint_{\mathbb{D}(r)} \Delta \alpha d \sigma
$$

so

$$
V^{\prime \prime}(r)-C V(r) \leq v^{\prime}(r)-C \int_{0}^{r} \frac{\rho}{r} v(\rho) d \rho=\frac{1}{2 \pi r} \iint_{\mathbb{D}(r)}(\Delta \alpha-C \alpha) d \sigma \leq 0 .
$$

Thus $\left(V^{\prime 2}(r)\right)^{\prime} \leq C\left(V^{2}(r)\right)^{\prime}$, so $V^{\prime}(r) \leq \sqrt{C} V(r)$, i.e., $\left(V(r) e^{\sqrt{C} r}\right)^{\prime} \leq 0$, which implies $V(r) \equiv 0$.

Lemma 9. Let $\phi_{1}$ and $\phi_{2}$ be Hopf differentials of harmonic mappings $h_{1}, h_{2}$ on $\mathbb{D}$ respectively, which satisfy that $\left|\phi_{1}(z)\right|=c>0$ and $\left|\phi_{1}\right|<\left|\phi_{2}\right|$. Then $\left|\mu_{1}(z)\right|<\left|\mu_{2}(z)\right|$ for all $z \in \mathbb{D}$.

Proof. Recall that

$$
\Delta \tau_{i}=\left(e^{2 \tau_{i}}-e^{-2 \tau_{i}}\right)\left|\phi_{i}\right|, \quad(i=1,2)
$$


where $\tau_{i}(z)=\frac{1}{2} \log \frac{1}{\left|\mu_{i}(z)\right|}(i=1,2)$. By Lemma 7 , we have $\left|\mu_{1}(z)\right| \leq\left|\mu_{2}(z)\right|$, so $\tau_{1}(z) \geq \tau_{2}(z)$. Let $\alpha(z)=\tau_{1}(z)-\tau_{2}(z)$. It satisfies $\alpha(z) \geq 0$ and

$$
\begin{aligned}
\Delta \alpha & =\left|\phi_{1}\right|\left(e^{2 \tau_{1}}-e^{-2 \tau_{1}}\right)-\left|\phi_{2}\right|\left(e^{2 \tau_{2}}-e^{-2 \tau_{2}}\right) \\
& \leq c\left(e^{2 \tau_{1}}-e^{2 \tau_{2}}+e^{-2 \tau_{2}}-e^{-2 \tau_{1}}\right) \\
& =c\left(e^{2 \tau_{1}}-e^{2 \tau_{2}}+\frac{e^{2 \tau_{1}}-e^{2 \tau_{2}}}{e^{2 \tau_{1}} e^{2 \tau_{2}}}\right) \\
& \leq 2 c\left(e^{2 \tau_{1}}-e^{2 \tau_{2}}\right) \\
& =2 c e^{2 \tau_{1}}\left(1-e^{-2 \alpha}\right) \\
& \leq 4 c e^{2 \tau_{1}} \alpha .
\end{aligned}
$$

Now we prove that $\alpha(z)>0$ on $\mathbb{D}$. Suppose there is a point $z_{0} \in \mathbb{D}$ such that $\alpha\left(z_{0}\right)=0$. Consider a disk $U\left(z_{0}, r\right)$ in $\mathbb{D}$ with the radius $r=\frac{1}{2} d\left(z_{0}, \partial \mathbb{D}\right)$. Since $\mu_{1}=\frac{\overline{\phi_{1}}}{e^{2 v_{1}}}$ and $\left|\phi_{1}(z)\right|=c>0$, the Beltrami coefficient $\mu_{1}(z) \neq 0$ for all $z \in \mathbb{D}$. So $\left|\mu_{1}(z)\right|$ has a positive lower bound on $U\left(z_{0}, r\right)$, which implies that $e^{2 \tau_{1}}$ has an upper bound on $U\left(z_{0}, r\right)$. Then there is $C>0$ which depends on $z_{0}$ such that $4 a e^{2 \tau_{1}}<C$, so we have $\Delta \alpha<C \alpha$ on $U\left(z_{0}, r\right)$. By Corollary $1 \alpha(z)=0$ for every point $z \in U$. By considering another point $z^{\prime} \in U\left(z_{0}, r\right)$ and continuing this procedure, we can obtain $\alpha(z)=0$ for all $z \in \mathbb{D}$. So we have $\alpha(z)=0$ for all $z \in \mathbb{D}$, which is impossible since $\left|\phi_{1}\right| \neq\left|\phi_{2}\right|$. So we have $\alpha(z)>0$ on $\mathbb{D}$, which yields $\left|\tau_{1}(z)\right|>\left|\tau_{2}(z)\right|$ for all $z \in \mathbb{D}$.

When the Hopf differential is equal to 0 , the harmonic mapping is conformal on $\mathbb{D}$. So the function $\gamma$ can be defined at $c=0$ with $\gamma(0)=0$.

Lemma 10. The function $\gamma(x)$ is continuous on $[0,+\infty)$.

Proof. Let $\phi_{1}$ and $\phi_{1}$ be two Hopf differentials whose Bers norms are bounded. Let $\mu_{i}=\frac{\overline{\phi_{i}}}{e^{2 v_{i}}}(i=1,2)$ be the corresponding Beltrami coefficient. It is known that

$$
\left\|\mu_{1}-\mu_{2}\right\|_{\infty} \rightarrow 0 \quad \text { as } \quad\left|\left\|\phi_{1}\right\|-\left\|\phi_{2}\right\|\right| \rightarrow 0 .
$$

For its proof one can see Proposition 14 of [4]. So the function $\gamma(x)$ is continuous on $[0, \infty)$.

Lemma 11. The function $\gamma$ satisfies

$$
\gamma(c) \geq \sqrt{1-\frac{4}{c}}
$$

for $c>4$. In particular, $\lim _{c \rightarrow+\infty} \gamma(c)=1$.

Proof. Let $\phi(z)$ be the Hopf differential of a quasiconformal harmonic mapping and $\mu(z)$ be the Beltrami coefficient. It is known (see [4]) that if $\|\mu\|_{\infty} \leq k<1$, the Hopf differential satisfies that

$$
\frac{|\phi|}{\rho^{2}} \leq \frac{1}{1-k^{2}} .
$$

Thus for $\phi=c$, we have $\frac{|\phi|}{\rho^{2}} \leq \frac{1}{1-\gamma(c)^{2}}$. In particular,

$$
\frac{|\phi|}{4} \leq \frac{1}{1-\gamma(c)^{2}}
$$


which yields

$$
\gamma(c) \geq \sqrt{1-\frac{4}{c}} .
$$

Proof of Theorem 1. Let $h(z)$ be a harmonic mapping whose Hopf differential is a constant $c>0$. By Lemmas 7 and 9 , the function

$$
\gamma(x): c \mapsto \mu(0),
$$

is strictly increasing from $(0,+\infty)$ to $(0,1)$. To prove it is a homeomorphism, we only need show $\gamma$ is continuous and surjective by Brouwer invariance of domain theorem. These are finished in Lemmas 10 and 11.

\section{Conclusions}

We have discussed a class of harmonic mappings whose Hopf differentials are constants. Let $h_{c}(z)$ be a harmonic mapping whose Hopf differential is a constant $c \in(0,+\infty)$. When $c$ is increasing from 0 to $+\infty$, it is proved that the vaule $\mu_{c}(0)$ is strictly increasing. Since the Beltrami coefficient $\mu_{c}(z)$ of $h_{c}(z)$ is radially symmetric and takes the maximum at $z=0$, the quasiconformal distortion $K_{c}$ of $h_{c}(z)$ is also strictly increasing.

Funding: This research received no external funding.

Conflicts of Interest: The author declares no conflict of interest.

\section{References}

1. Li, P.; Tam, L.-F. Uniqueness and regularity of proper harmonic maps. Ann. Math. 1993, 137, 167-201. [CrossRef]

2. Li, P.; Tam, L.-F. Uniqueness and regularity of proper harmonic maps II. Indiana Univ. Math. J. 1993, 42, 591-635. [CrossRef]

3. Markovic, V. Harmonic maps and the Schoen conjecture. J. Am. Math. Soc. 2017, 30, 799-817. [CrossRef]

4. Wan, T. Constant mean curvature surface, harmonic maps, and universal Teichmüller space. J. Differ. Geom. 1992, 35, 643-657. [CrossRef]

5. Wolf, M. The Teichmüller theory of harmonic maps. J. Differ. Geom. 1989, 29, 449-479. [CrossRef]

6. Tam, L.-F.; Wan, T. Harmonic diffeomorphisms between Cartan- Hadamard surfaces with prescribed Hopf differentials. Commun. Anal. Geom. 1994, 2, 593-625. [CrossRef]

7. Yau, S.T. Harmonic functions on complete Riemannian manifolds. Commun. Pure Appl. Math. 1975, 28, 201-228. [CrossRef]

8. Cheng, S.Y.; Yau, S.T. Differential equations on Riemannian manifolds and their geometric applications. Commun. Pure Appl. Math. 1975, 28, 333-354. [CrossRef]

9. Omori, H. Isometric immersions of Riemannian manifolds. J. Math. Soc. Jpn. 1967, 19, 205-214. [CrossRef]

10. Heinz, E. On certain nonlinear elliptic differential equations and univalent mappings. J. Anal. Math. 1956, 5, 197-272. [CrossRef]

(C) 2020 by the authors. Licensee MDPI, Basel, Switzerland. This article is an open access article distributed under the terms and conditions of the Creative Commons Attribution (CC BY) license (http://creativecommons.org/licenses/by/4.0/). 